\title{
Cab transfer process improvement at an automotive manufacturing facility
}

\begin{abstract}
This project is related to the improvement of cab transfer process from Painted Body Storage (PBS) area to General Assembly (GA) line. The objectives of this project are to eliminate the root cause of cab defect and to streamline the transfer process with the reduction of process cycle time. From the current process study, it is found that the lifting method causes the quality defect to the cab and the usage of different type of trolley between PBS and GA contribute to the high cycle time. The new process design has been introduced from the findings with the use of towing method and the use of common trolley for both PBS area and GA line. With the new and improved cab transfer process, the cab changing process can be eliminated. Hence, the number of operator required has been reduced, from two operators to only one operator. A trial run has been conducted with a prototype trolley and a forklift. The objectives of this project are fulfilled with the new towing method that eliminate the quality defect and the use of common trolley for both PBS and GA line resulting new cycle time of 7.13 minutes per unit from 9.77 minutes per unit. Further studies are needed for commissioning and monitoring to the new cab transfer process with the actual trolley and tow tug or buggy.
\end{abstract}

Keyword: Improvement; Kaizen 